\title{
NEW CALIBRATION OF THE MICROMETEOROID FLUX ON EARTH
}

\author{
G. Cremonese ${ }^{1}$, P. Borin ${ }^{1}$, E. Martellato ${ }^{2}$, F. Marzari $^{3}$, and M. Bruno \\ ${ }^{1}$ Istituto Nazionale di Astrofisica-Astronomical Observatory of Padova, 35122 Padova, Italy \\ 2 SRE-SM, European Space Agency-ESTEC, Keplerlaan 1, 2201 AZ Noordwijk, The Netherlands \\ 3 Department of Physics, University of Padova, 35131 Padova, Italy \\ ${ }^{4}$ Department of Mineralogical and Petrological Science, University of Torino, 10125 Torino, Italy \\ Received 2011 November 21; accepted 2012 March 16; published 2012 April 2
}

\begin{abstract}
Mass accretion rate on Earth is an important tool to discriminate the extraterrestrial nature of particles or isotopes found in different environments on the ground. In this context, the knowledge of the micrometeoroid flux arriving in our atmosphere is a key parameter and it needs to be calibrated. We provide a new calibration of the flux of submillimeter particles impacting the Earth in the mass range from $10^{-9}$ to $10^{-4} \mathrm{~g}$, derived by computing a specific scaling law for impact craters on the Long Duration Exposure Facility (LDEF). We use the hydrocode iSALE to calculate the outcome of impacts on LDEF, adopting realistic impact velocities for dust particles derived from the numerical integration of their trajectories assuming either asteroidal or cometary origin. We estimate a particle mass accretion rate of $(7.4 \pm 1.0) \times 10^{6} \mathrm{~kg} \mathrm{yr}^{-1}$ if the Main Belt is assumed as the major source of dust, while it reduces to $(4.2 \pm 0.5) \times 10^{6} \mathrm{~kg} \mathrm{yr}^{-1}$ if cometary dust dominates. These values agree with the estimates provided by independent measurements made on ice core and ocean sediments and based on the abundance of some elements in the samples.
\end{abstract}

Key words: Earth - meteorites, meteors, meteoroids - methods: numerical

\section{INTRODUCTION}

Earth's meteoritic mass accretion rate plays an important role in many contexts. Meteoroids entering Earth's upper atmosphere deposit meteoritic matter there, a process which has important consequences for the aeronomy of the upper mesosphere and lower ionosphere. It provides important input on the analysis of the deep-sea sediments and ice core samples in terms of some element abundance and it may help to quantify the meteoroid collision hazards for spacecraft. The observations and measurements of meteoroids in our atmosphere could also be important for calibrating the models of the terrestrial planet environment (Borin et al. 2010). We determined the mass flux and size distribution of extraterrestrial micrometeoroids in the submillimeter radius size range of $5-250 \mu \mathrm{m}$ from accurate measurements of the dimensions of all the hypervelocity impact craters collected on the space-facing end of the gravity-gradientstabilized Long Duration Exposure Facility (LDEF) satellite (Love \& Brownlee 1993). According to Flynn (2002), more than $80 \%$ of all the incoming mass of meteoroids entering Earth's atmosphere is concentrated in the mass range $10^{-7}$ to $10^{-3} \mathrm{~g}$. In our model, we considered a slightly larger range, $10^{-9}$ to $10^{-4} \mathrm{~g}$, in order to include possible uncertainty. The first step for translating the crater data on LDEF into a flux estimate is to interpret the crater diameters in terms of projectile size. Love \& Brownlee (1993) adopted a semiempirical scaling law derived from laboratory experiments that were performed in a range of velocities significantly different with respect to the impact speeds of impacting meteroids. To improve the analysis of LDEF data, we did a specific exploration of the relation between crater diameter and projectile size with the hydrocode iSALE. In the numerical modeling, the ratio between the depth and diameter of the craters of 0.527, accurately measured on LDEF (Love \& Brownlee 1993), has been considered as an important constraint for the iSALE simulations. The range of impact velocities to be used in the hydrocode simulations has been derived using the dynamical model of dust particle orbital evolution described in Borin et al. (2009). The orbits of dust particles released either from collisions between Main Belt Asteroids (MBAs) or from cometary activity on Jupiter Family Comets (JFCs) are numerically integrated, including the gravitational attraction of all planets and the effects of solar radiation and wind forces. The impact velocity on the Earth of the particles is computed for both sources, giving two significantly different average values. For asteroidal dust particles, we obtain an impact speed of about $18.6 \mathrm{~km} \mathrm{~s}^{-1}$ while it rises to $29.0 \mathrm{~km} \mathrm{~s}^{-1}$ for JFCs. Both these values differ from those adopted by Love \& Brownlee (1993) and by those adopted in the derivation of the experimental scaling law adopted by Love \& Brownlee (1993). This fully justifies a re-analysis of the LDEF data with a more reliable scaling law based on a better impact velocity estimate and on hydrocode simulations that also account for the different porosity and composition of the dust grains depending on their origin (asteroidal or cometary).

\section{2. iSALE MODELING}

To simulate the impact process, we used iSALE shock physics code, which represents an extension and an improvement of the SALE hydrocode developed by Amsden et al. (1980), and includes an elasto-plastic constitutive model, fragmentation models, various equations of state (EoSs), the implementation of multiple materials, and a novel porosity compaction model, the $\varepsilon-\alpha$-model (Melosh et al. 1992; Ivanov et al. 1997; Collins et al. 2004; Wünnemann et al. 2006). The code is well tested against laboratory experiments at low and high strain rates (Wünnemann et al. 2006) and other hydrocodes (Pierazzo et al. 2008). We assumed the projectile radius range to be from 5 to $250 \mu \mathrm{m}$, with a step of $20 \mu \mathrm{m}$. Under the assumption that the average flight angle of the LDEF facility was $45^{\circ}$ with respect to the local zenith, following Love \& Brownlee (1993), we adopt vertical impact components of 13.2 and $20.5 \mathrm{~km} \mathrm{~s}^{-1}$, respectively. The measured craters from which the meteoroids flux was derived have been collected in a $5.66 \mathrm{~m}^{2}$ 


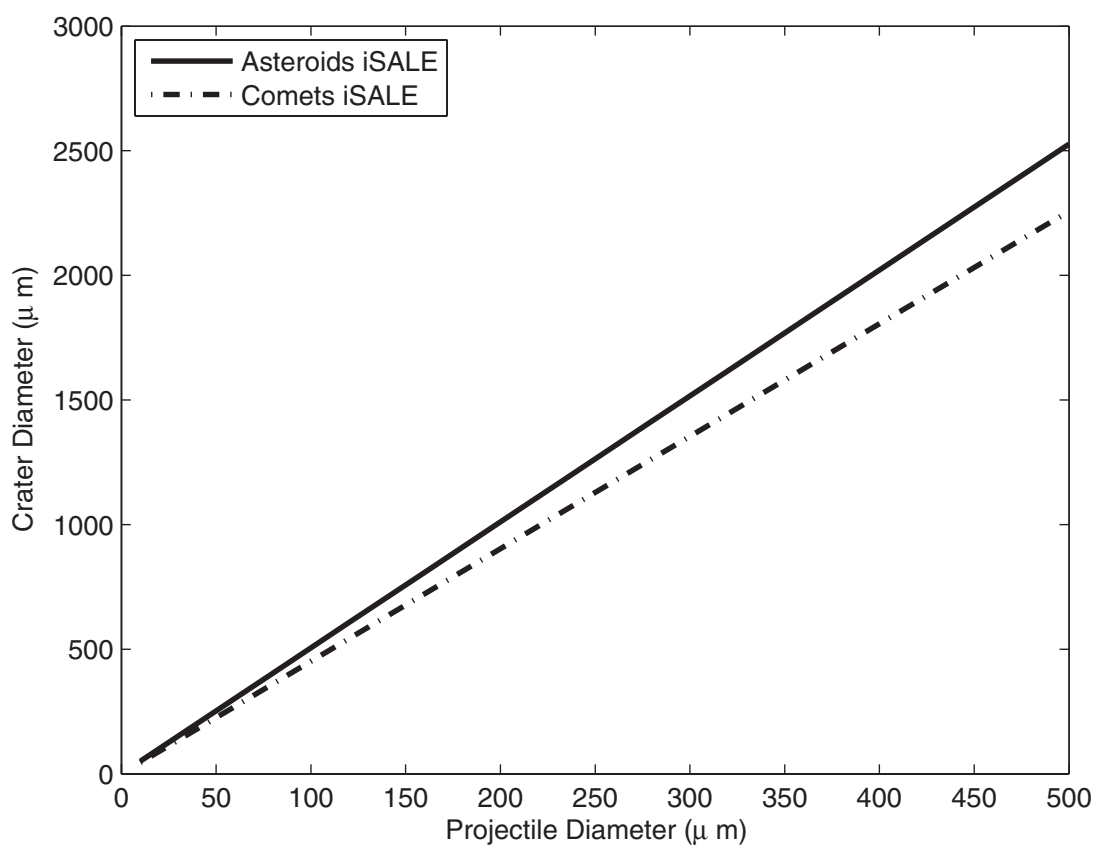

Figure 1. Scaling law obtained from iSALE simulations.

thermal control panel surface exposed to space for 5.77 years at altitudes ranging from 480 to $331 \mathrm{~km}$ (Love \& Brownlee 1993). To model the impact events, we considered an Eulerian mesh large enough that the target could be considered "infinite" with respect to the final expected crater, and a gravity field of $8.6963 \mathrm{~m} \mathrm{~s}^{-2}$. The target was made up by a sheet of aluminum 6061-T6 alloy, which is described by the Tillotson Equation of State (see Table AII.3 in Melosh 1989) to account for bulk deformations and the Johnson-Cook model (see Table 4 in Rule 1997) to account for deviatoric deformations. All the projectiles have been assumed basalt spheres at 50 cells per projectile radius of resolution, having a density of $2.86 \mathrm{~g} \mathrm{~cm}^{-3}$. The basalt is chosen to be representative of a general brittle rock behavior. The thermodynamic state of basalt is described by the ANEOS-based tabular material EoSs. The strength model implemented in iSALE is described in Collins et al. (2004), who used the Lundborg (1968) approximation to account for the effect of pressure on yield strength for intact rock, and a Coulomb dry-friction law in the case of totally fragmented rock material. The constitutive parameters adopted are the following: $Y_{0}$ (cohesion for intact material) $=10 \mathrm{MPa}, \mu_{i}$ (coefficient of internal friction $)=0.8, Y_{M}$ (von Mises plastic limit $)=$ $1 \mathrm{GPa}, Y_{d}$ (cohesion for damaged material) $=0.01 \mathrm{MPa}, \mu_{d}$ $($ coefficient of friction $)=0.6$. We have run the simulations even taking into account a much lower cohesion value of $10 \mathrm{kPa}$ (Trigo-Rodriguez \& Llorca 2006), but the results are not significantly affected. To differentiate the two types of test particles, we set a different porosity: $25 \%$ for asteroidal dust, giving a density of $2.14 \mathrm{~g} \mathrm{~cm}^{-3}$, and $70 \%$ for cometary dust yielding a density of $0.86 \mathrm{~g} \mathrm{~cm}^{-3}$. These values are consistent with recent literature on the expected density and porosity of meteoroids (Greenberg \& Aigen 1999; Greenberg 2000; Rietmeijer \& Nuth 2000; Sykes et al. 2004). As a further test, we also assumed a higher porosity for the cometary dust particles of $90 \%$ yielding a density of $0.286 \mathrm{~g} \mathrm{~cm}^{-3}$. This is an extreme value for the density according to Blum et al. (2006). In our simulations, the formation time of the crater ranges from a fraction of $\mu \mathrm{s}$ to few $\mu \mathrm{s}$. The resulting average ratio between depth and diameter is 0.582 for asteroids and 0.529 for comets.
Both these values are in very good agreement with the average ratio measured on LDEF of 0.527 (Love \& Brownlee 1993). We must also acknowledge that these ratios depend on the precision of the hydrocode simulations which, according to code validation against laboratory experiments (10), is $3 \%-4 \%$ in radius and $12 \%$ in depth. The results of the iSALE simulations provide a new scaling law (Figure 1) that is significantly different from that used by Love \& Brownlee (1993), suggesting that they systematically overestimated the projectile size for any given crater diameter.

\section{RESULTS}

From the new scaling laws specific for asteroidal or cometary dust grains, we have re-analyzed the LDEF data and computed updated values of the dust flux. In Figures 2 and 3, we show the cumulative and differential flux of dust particles on the Earth as a function of the grain mass. By comparing our curves with that of Love \& Brownlee (1993) a noticeable difference stands out. The flux estimated with the scaling law adopted in Love \& Brownlee (1993) has a peak value that is more than three times higher than the one we obtained for either asteroidal or cometary dust. It has to be noted that the estimated flux for dust coming from JFCs is lower than the asteroidal counterpart, since cometary dust has a higher impact velocity and this leads to smaller projectiles for the same impact energy. This is confirmed in Figures 2 and 3, where the mass range for cometary and asteroidal dust particles is different with the asteroidal mass range shifted toward larger values.

From the curves of Figure 3 we have re-calculated the integral of the mass flux, and we obtain a value for the total mass accreted by the Earth of $(4.2 \pm 0.5) \times 10^{6} \mathrm{~kg} \mathrm{yr}^{-1}$ for comets and of $(7.4 \pm 1.0) \times 10^{6} \mathrm{~kg} \mathrm{yr}^{-1}$ for asteroids, where the sigma have been derived from the $4 \%$ uncertainty of the hydrocode in the diameter determination and the consequent variation of the curves. In the extreme case where a porosity of $90 \%$ is assumed for the cometary dust, we obtain a total mass of $14.3 \times 10^{6} \mathrm{~kg} \mathrm{yr}^{-1}$. All these values of total mass accreted are significantly lower than that estimated by Love \& Brownlee 


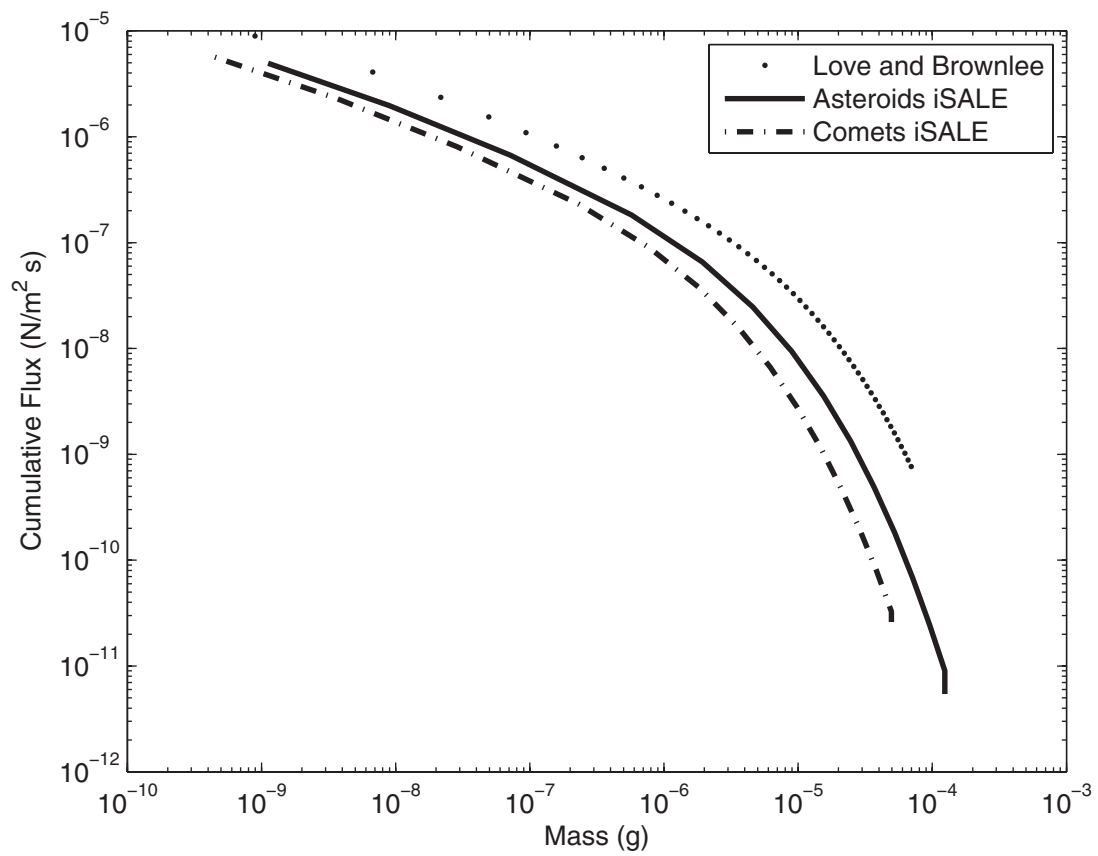

Figure 2. Cumulative flux of dust grains derived interpreting the LDEF data with the iSALE scaling laws and adopting the impact speeds derived by our dynamical model. The flux is expressed as the number of particles $N$ per $\mathrm{m}^{-2} \mathrm{~s}^{-1}$. The curves derived with the iSALE scaling laws are compared to the original flux computed by Love \& Brownlee (1993).

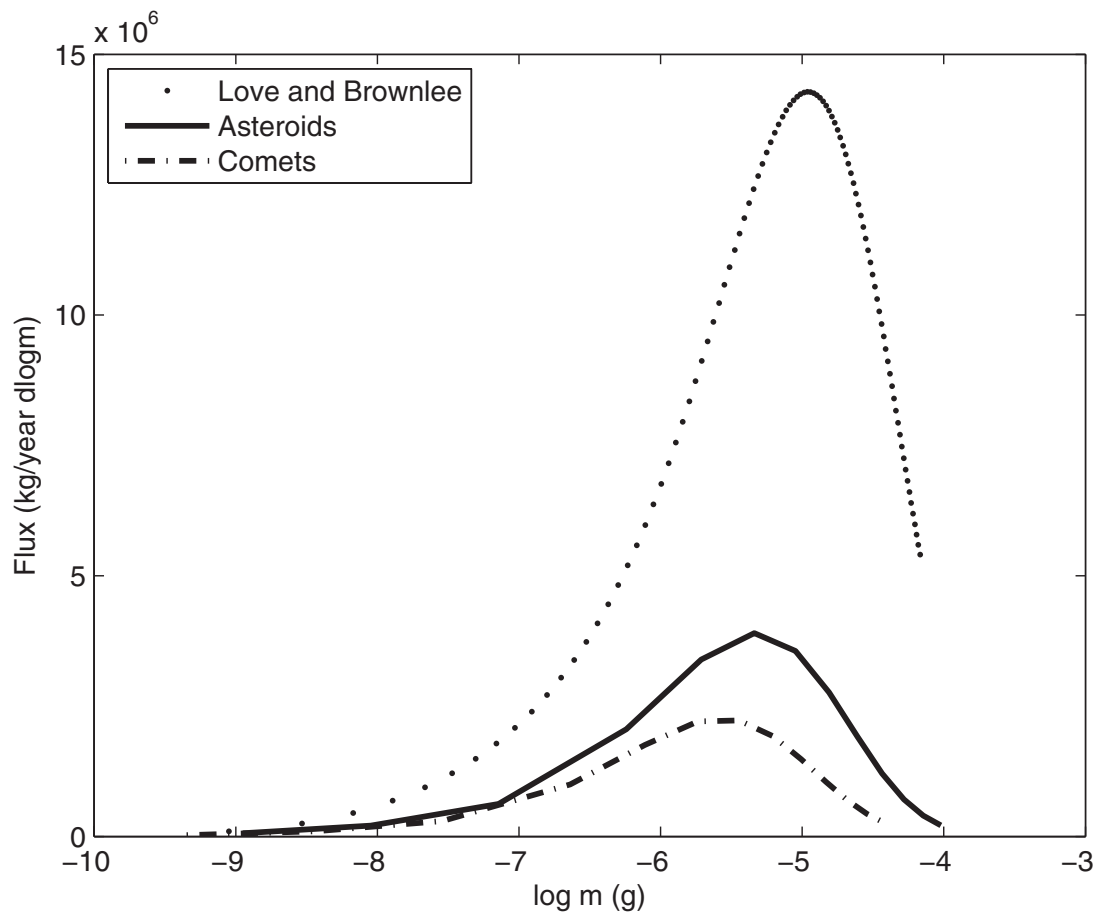

Figure 3. Mass accretion rate of micrometeoroids on Earth according to our model and to Love \& Brownlee (1993).

(1993) $\left((40 \pm 20) \times 10^{6} \mathrm{~kg} \mathrm{yr}^{-1}\right)$ even after the revision proposed by Mathews et al. (2001) is applied $\left((25 \pm 12.5) \times 10^{6} \mathrm{~kg} \mathrm{yr}^{-1}\right)$. An error can be evaluated for our estimates taking into account that there is a $4 \%$ uncertainty in the diameter determination from the hydrocode simulations.

Our values for the mass accretion rate are in agreement with estimates obtained with different methods (see Table 1 of Karner et al. 2003). In particular, Mathews et al. (2001), on the basis of radar observations of micrometeors, derived a value of (1.6-2.3) $\times 10^{6} \mathrm{~kg} \mathrm{yr}^{-1}$, and in order to reconcile their findings with the mass accretion rate computed by Love \& Brownlee
(1993), they revised the Love \& Brownlee (1993) calculations assuming a much higher impact speed for the dust particles using the value $50 \mathrm{~km} \mathrm{~s}^{-1}$. In this way, they derive a value of $1.8 \times 10^{6} \mathrm{~kg} \mathrm{yr}^{-1}$ from the data analyzed by Love \& Brownlee (1993). However, according to our dynamical model, an impact speed of $50 \mathrm{~km} \mathrm{~s}^{-1}$ is by far too high, almost a factor of two larger than the value expected for cometary dust $\left(29.0 \mathrm{~km} \mathrm{~s}^{-1}\right)$, and cannot be adopted. Grün et al. (1985) provided another estimate, based on impact craters and meteoroid flux, of $14.6 \times 10^{6} \mathrm{~kg} \mathrm{yr}^{-1}$ that is not far from our estimate considering the uncertainties. However, it is worth noticing that previous works based on the 
analysis of impact craters and meteoroid dynamics did not take into account asteroids or comets as different sources of dust release.

\section{CONCLUSIONS}

The data recoiled by LDEF on the flux of dust particles on the Earth have been re-evaluated adopting a better estimate of the grain impact velocity and more reliable scaling laws. In this Letter, we consider both dust coming from the MBA and from JFCs, which are the most plausible candidates for refilling with dust the Earth environment (Dermott et al. 2002; Nesvorny et al. 2010). In the two cases, the scaling law giving the projectile size from the crater diameter will differ because the grains have a different porosity and structure. In addition, for the same impact energy, the collision velocities are different due to the different dynamical source. The scaling laws we find for asteroids and comets significantly depart from that used by Love \& Brownlee (1993), leading to significantly different curves describing the flux as a function of the dust grain mass and a different total mass accretion rate. Our values are approximately 10 times lower and are in better agreement with other independent estimates based on different methodologies.

The dust concentrates extracted from Greenland Ice Sheet Project 2 ice core samples by Karner et al. (2003) allowed us to estimate the rate of extraterrestrial accretion for particles in the size range $0.45-20 \mu \mathrm{m}$ of $(0.22 \pm 0.11) \times 10^{6} \mathrm{~kg} \mathrm{yr}^{-1}$, one order of magnitude lower than that estimated by Love \& Brownlee (1993) in the same range $\left(5 \times 10^{6} \mathrm{~kg} \mathrm{yr}^{-1}\right)$. They extrapolated their results to larger particles, up to $4 \mathrm{~cm}$, by using both

1. the particle distribution of Grün et al. (1985) with Monte Carlo simulations, in order to determine an upper limit of $6.25 \times 10^{6} \mathrm{~kg} \mathrm{yr}^{-1}$, and

2. the Love \& Brownlee (1993) particle distribution, $12.5 \times$ $10^{6} \mathrm{~kg} \mathrm{yr}^{-1}$.

Karner et al. (2003) emphasized that the results of Love \& Brownlee (1993) would imply that they were missing $96 \%$ of the extraterrestrial material because of undersampling, suggesting that the Love \& Brownlee (1993) distribution function may represent a systematic overestimate of particle masses as a consequence of the underestimation of impact velocity. A similar problem was also pointed out by Taylor et al. (1998) in their collection of cosmic spherules at the South Pole. In their case, to comply with the Love \& Brownlee (1993) flux they assumed that $96 \%$ of the mass would have been ablated during atmospheric entry. Assuming that the size range considered by Karner et al. (2003) could account for less than $10 \%$ of the incoming mass of meteoroids entering Earth's atmosphere (Flynn 2002), our particle distribution in that size range yields $<0.42 \times 10^{6} \mathrm{~kg} \mathrm{yr}^{-1}$ and $<0.74 \times 10^{6} \mathrm{~kg} \mathrm{yr}^{-1}$ for comets and asteroids, respectively. Our mass accretion rate would then agree with even the Taylor results of $(1.6 \pm 0.3) \times 10^{6} \mathrm{~kg} \mathrm{yr}^{-1}$, within the uncertainties, without assuming a large percentage of meteoroids ablated.

Analysis of ocean sediment samples has been used to estimate the mass accretion rate on Earth, but the values determined have large uncertainties or have been revised in following works. Indeed, the value yielded by the IR measurements of Kyte \& Wasson (1996) has been revised downward by PeuckerEhrenbrink (1996) to $(30 \pm 15) \times 10^{6} \mathrm{~kg} \mathrm{yr}^{-1}$, which is higher compared to our results but still significantly lower than the value given by Love \& Brownlee (1993). It can be considered in agreement with our values if the large uncertainty in their estimate is taken into account. We found a better agreement with the excess ${ }^{3} \mathrm{He}$ in ocean sediments. The studies of Farley \& Patterson (1995) and Marcantonio et al. (1999) yielded measurements of ${ }^{3} \mathrm{He}$ very consistent with Karner et al. (2003), and assuming that the $\mathrm{He}$ is correlated with Interplanetary Dust Particle surface area the estimated mass flux of extraterrestrial material to the deep ocean is $7.5 \times 10^{6} \mathrm{~kg} \mathrm{yr}^{-1}$, which is very similar to our value obtained for asteroids.

We gratefully acknowledge the developers of iSale, including Kai Wunnemann, Gareth Collins, Boris Ivanov, Jay Melosh, and Dirk Elbesbausen (see www.iSALE-code.de).

\section{REFERENCES}

Amsden, A. A., Ruppel, H. M., \& Hirt, C. W. 1980, Sale: A Simplified ALE Computer Program for Fluid Flows at All Speeds, Los Alamos National Laboratories, Report LA-8095

Blum, J., Schrapler, R., Davidson, B. J. R., \& Trigo-Rodriguez, J. M. 2006, ApJ, 652, 1768

Borin, P., Bruno, M., Cremonese, G., \& Marzari, F. 2010, A\&A, 517, A89

Borin, P., Cremonese, G., Marzari, F., Bruno, M., \& Marchi, S. 2009, A\&A, 503,259

Collins, G. S., Melosh, H. J., \& Ivanov, B. A. 2004, Meteorit. Planet. Sci., 39, 217

Dermott, S. F., Durda, D. D., Grogan, K., \& Kehoe, T. J. J. 2002, Asteroids III (Tucson, AZ: Univ. Arizona Press), 423

Farley, K. A., \& Patterson, D. B. 1995, Nature, 378, 600

Flynn, G. J. 2002, in Meteors in the Earth Atmosphere, ed. E. Murad \& I. P. Wiliamseds (Cambridge: Cambridge Univ. Press), 77

Greenberg, J. M. 2000, Earth Moon Planets, 82, 313

Greenberg, J. M., \& Aigen, L. 1999, Space Sci. Rev., 90, 149

Grün, E., Zook, H. A., Fechtig, H., \& Giese, R. H. 1985, Icarus, 62, 244

Ivanov, B. A., Deniem, D., \& Neukum, G. 1997, Int. J. Impact Eng., 20, 411

Karner, D. K., Levine, J., Muller, R. A., et al. 2003, Geochim. Cosmochim. Acta, 67, 751

Kyte, F. T., \& Wasson, J. T. 1986, Science, 232, 1225

Love, S. G., \& Brownlee, D. E. 1993, Science, 262, 550

Lundborg, N. 1968, Int. J. Rock Mech. Min. Sci., 5, 427

Marcantonio, F., Turekian, K. K., Higgins, S., et al. 1999, Earth Planet. Sci. Lett., 170, 157

Mathews, J. D., Janches, D., Meisel, D. D., \& Zhou, Q. H. 2001, Geophys. Res. Lett., 28, 101929

Melosh, H. J. 1989, Impact Cratering: A Geologic Process (New York: Oxford Univ. Press), 245

Melosh, H. J., Ryan, E. V., \& Asphaug, E. 1992, J. Geophys. Res., 97, 14

Nesvorny, D., Jenniskens, P., Levison, H. F., et al. 2010, ApJ, 713, 816

Peucker-Ehrenbrink, B. 1996, Geochim. Cosmochim. Acta, 60, 3187

Pierazzo, E., Artemieva, N., Asphaug, E., et al. 2008, Meteorit. Planet. Sci., 43, 1917

Rietmeijer, F. J. M., \& Nuth, J. A., III 2000, Earth Moon Planets, 82, 325

Rule, W. K. 1997, Int. J. Impact Eng., 5, 797

Sykes, M. V., Grün, E., Reach, W. T., \& Jenniskens, P. 2004, in Comets II, ed. M. Festou, H. U. Keller, \& H. A. Weaver (Tucson, AZ: Univ. Arizona Press), 677

Taylor, S., Lever, J. H., \& Harvey, R. P. 1998, Nature, 392, 899

Trigo-Rodriguez, J. M., \& Llorca, J. 2006, MNRAS, 372, 655

Wünnemann, K., Collins, G. S., \& Melosh, H. J. 2006, Icarus, 180, 514 